\title{
BMJ Open Patients' preferences for health insurance coverage of new technologies for treating chronic diseases in China: a discrete choice experiment
}

\author{
Jinsong Geng (D) , ${ }^{1}$ Xiaowei Chen, ${ }^{1,2}$ Haini Bao, ${ }^{1}$ Danmin Qian, ${ }^{1}$ Yuting Shao, ${ }^{3}$ \\ $\mathrm{Hao} \mathrm{Yu}$ (1) ${ }^{4}$
}

To cite: Geng J, Chen X, Bao H, et al. Patients' preferences for health insurance coverage of new technologies for treating chronic diseases in China: a discrete choice experiment. BMJ Open 2020;10:e038051. doi:10.1136/ bmjopen-2020-038051

- Prepublication history for this paper is available online. To view these files, please visit the journal online (http://dx.doi. org/10.1136/bmjopen-2020038051).

Received 27 February 2020 Revised 31 August 2020 Accepted 01 September 2020

Check for updates

(C) Author(s) (or their employer(s)) 2020. Re-use permitted under CC BY-NC. No commercial re-use. See rights and permissions. Published by BMJ.

${ }^{1}$ Department of Medical Informatics, Nantong University Medical School, Nantong, Jiangsu, China

${ }^{2}$ Library and Reference Department, Zhejiang University School of Medicine First Affiliated Hospital, Hangzhou, Zhejiang, China

${ }^{3}$ Department of Ophthalmology, Tongji University School of Medicine, Shanghai, China ${ }^{4}$ Department of Population Medicine, Harvard Medical School and Harvard Pilgrim Health Care Institute, Boston, Massachusetts, USA

Correspondence to Professor Jinsong Geng; gjs@ntu.edu.cn

\section{ABSTRACT}

Objectives Our study aimed to inform insurance decisionmaking in China by investigating patients' preferences for insurance coverage of new technologies for treating chronic diseases.

Design We identified six attributes of new medical technologies for treating chronic diseases and used Bayesian-efficient design to generate choice sets for a discrete choice experiment (DCE). After conducting the DCE, we analysed the data by mixed logit regression to examine patient-reported preferences for each attribute. Setting The DCE was conducted with patients in six tertiary hospitals from four cities in Jiangsu province. Participants Patients aged 18 years or older with a history of diabetes or hypertension and taking medications regularly for more than 1 year were recruited $(n=408)$. Results The technology attributes regarding expected gains in health outcomes from the treatment, high likelihood of effective treatment and low incidence of serious adverse events were significant, positive predictors of choice by the study patients $(p<0.01)$. The out-of-pocket cost was a significant, negative attribute for the entire study sample $(\beta=-0.258, p<0.01)$ and for the patients with Urban-Rural Residents Basic Medical Insurance (URRBMI) $(\beta=-0.511, p<0.01)$, but not for all the patients with Urban Employees Basic Medical Insurance (UEBMI) $(\beta=-0.071, p>0.05)$. The severity of target disease was valued by patients with lower EQ-5D-5L index value as well as URRBMI enrollees.

Conclusions Patients highly valued the health benefits and risks of new technologies, which were closely linked to their feelings of disease and perceptions of healthrelated quality of life. However, there existed heterogeneity in preferences between URRBMI and UEBMI patients. Further efforts should be made to reduce the gap between insurance schemes and make safe and cost-effective new technologies as a priority for health insurance reimbursement.

\section{INTRODUCTION}

Non-communicable chronic diseases (chronic diseases) are health conditions or diseases with long-term accumulation, nonself-healing and difficult to cure. Nowadays, the prevalence and mortality of chronic
Strengths and limitations of this study

- Our study provides evidence regarding patients preferences for insurance coverage of new technologies for treating chronic diseases and will be helpful for applying a patient-centred approach to policy-making.

- The discrete choice experiment is a rigorous method that enables us to identify differential preferences among chronic disease patients by type of social health insurance and by the level of health-related quality of life in China.

- The Bayesian-efficient design was used to improve the statistical efficiency of the choice design, and a blocking technique was used to increase the response efficiency of patients.

- Since our sample was from a wealthy province in China, future studies of nationally representative samples are needed.

- While this study focussed on hypertension and diabetes, two of the most prevalent chronic diseases, future studies need to examine other types of chronic diseases.

diseases are on the rise around the world. ${ }^{1}$ Chronic diseases present a particularly daunting challenge to China. It was estimated that among Chinese adults aged 35 to 75 years, nearly half of them had hypertension. $^{2}$ The overall prevalence of diabetes in Chinese adults was about $10.9 \% .^{3}$ Furthermore, comorbidities are highly prevalent among patients with chronic diseases, which harm the patient's quality of life and impede the efficacy of treatment. ${ }^{4-6}$ Chronic diseases lead to a heavy financial burden on patients' families and health insurance programmes. It was estimated that the total economic burden associated with chronic diseases in China over the period 2010 to 2030 could be as high as US $\$ 16$ trillion (measured in 2010 US dollars (US\$) $).^{7}$ Further adding to the challenges to China's health insurance programmes' 
financing capacity, new technologies for treating chronic diseases continue to enter the market, which can be very expensive and contribute to rising healthcare costs. Deciding on which new technology to cover and by which insurance programme has become a key issue facing policymakers in China in the context of universal health insurance coverage.

As part of its goal of providing timely, acceptable and affordable basic healthcare of appropriate quality to its residents, China successfully achieved universal health insurance coverage in 2011, increasing demand for and expenditures on healthcare. China's total health expenditures grew at an average annual rate of $12.2 \%$ from 2008 to 2017, which was much higher than its gross domestic product's average annual growth rate $(8.1 \%) .{ }^{8}$ In recent years, Chinese policymakers have struggled to keep a balance between expenditure control and meeting patients' demand for healthcare, including the demand for new technologies by patients with chronic diseases. China's National Healthcare Security Administration is promoting the health insurance payment based on diagnosis-related groups (DRGs), a patient classification for standardising payment in the national health insurance schemes. Consequently, medical fees and insurance payments will be determined in accordance with the DRG classification, which includes chronic diseases such as diabetes and hypertension. ${ }^{9}$

After China had reached universal health insurance coverage, there were still considerable disparities in benefit coverage and reimbursement ratio among the three major public insurance programmes that together covered more than $95 \%$ of Chinese people, including New Rural Cooperative Medical Scheme (NRCMS), Urban Residents Basic Medical Insurance (URBMI) and Urban Employees Basic Medical Insurance (UEBMI). For further details on differences in health insurance eligibility, premiums and benefits among the three programmes, see Yu 2015. ${ }^{10}$ Generally speaking, UEBMI has the best benefits package and the lowest out-of-pocket cost among the three public insurance programmes, ${ }^{11} 12$ and UEBMI enrollees had a higher likelihood of healthcare utilisation. ${ }^{13}$ To improve administrative efficiency and reduce inequality in insurance benefits, the China State Council issued a policy in January 2016 merging the NRCMS and URBMI to form the Urban-Rural Residents Basic Medical Insurance (URRBMI) ${ }^{14}$ While the newly formed URRBMI helped equalise insurance benefits between urban and rural residents, gaps remained between URRBMI and UEBMI. For example, according to the 2018 statistical bulletin issued by China's National Healthcare Security Administration, the average per capita inpatient hospitalisation cost was Chinese Yuan (CNY) 11181 (about US\$1704) for UEBMI enrollees and CNY 6577 (about US\$1003) for URRBMI enrollees. ${ }^{15}$ The average inpatient reimbursement ratio for UEBMI enrollees was $71.8 \%$, and the reimbursement ratio for URRBMI enrollees was $56.1 \% .{ }^{15}$ Even among the UEBMI enrollees, the insurance benefit is not comparable, as some of the enrollees enjoy civil servant subsidies. ${ }^{16}$ For example, if the medical expenditure exceeds the ceiling of health insurance reimbursement, outpatients and inpatients that enjoy civil servant subsidies may still be subsidised by $70 \%$ and $80 \%$ respectively for the exceeding parts. ${ }^{17}$ Whereas disparities in China's insurance programmes and patients' utilisation of healthcare have been well-documented in the literature, no studies have yet examined whether patients' preferences for new medical technology vary by type of insurance. This study aimed to fill the gap.

Patient-reported outcome measures, such as healthrelated quality of life (HRQoL), are useful for understanding the impact of the disease on their functional status and well-being. ${ }^{18}$ EQ-5D series are among the most widely used multi-attribute utility instruments to measure HRQoL. EQ-5D-5L uses a health-state classification system that defines health in five areas, ${ }^{19}$ has been proved to have the validity and discriminatory power to measure HRQoL in patients with chronic diseases. ${ }^{20-22}$ Although HRQoL is an essential measure of health status to inform public health and health policy, whether patients' preferences on reimbursement differ from HRQoL remains unclear.

Eliciting patients' preferences and involving patients in health insurance decision-making can be helpful to increase the satisfaction of patients and is an integral part of patient-centred care. This is defined as providing care that is respectful of, and responsive to individual patient's preferences, needs and values. ${ }^{23}$ Although the patient-centred approach and value-based care have long been advocated in China, there is still a lack of evidence from patients reflecting their preferences to inform health insurance coverage decision-making. This study adds new information to the literature by conducting a discrete choice experiment (DCE), which is an attributebased method to measure the preferences and tradeoffs of respondents and becomes a recognised scientific approach to elicit preferences. ${ }^{24}$ Prior research showed that DCE was fruitful and reliable to improve healthcare decision-making effectively. ${ }^{25}$ Our DCE focussed on chronic disease patients, and its goal was to support evidence-informed insurance policy-making in China. Specifically, we used the DCE data to test the following hypotheses: (1) new technology attributes regarding health benefits are more important than other attributes for health insurance coverage and (2) patients' preferences differ by type of disease and type of insurance.

\section{METHODS}

\section{Identification of technology attributes and levels}

Our DCE design, implementation and analysis followed the user guide developed jointly by the World Bank, WHO and US Agency for International Development. ${ }^{26}$ We used a three-step approach to complete the preliminary stage of DCE, which aimed to identify and define the attributes and levels of new medical technologies. First, a systematic review was conducted to select attributes 
that were often used in multi-criteria decision analyses of health insurance decision-making. The systematic review was performed according to the framework for evidencebased decision-making as defined by 'Evidence and Value: Impact on DEcisionMaking' (EVIDEM). ${ }^{27}$ We found that the most commonly mentioned dimensions were comparative outcomes (effectiveness, safety/tolerability), economic consequences (costs and cost-effectiveness), needs of new technologies (severity of target disease, size of the affected population, unmet needs related to the already reimbursed technologies), and knowledge of new technologies (quality of evidence, expert consensus/clinical practice guideline) ${ }^{28}$

Second, both focus group discussions with physicians and expert consultation were carried out to determine the attributes used in our research. There was no consensus among physicians about the criteria to determine the level of attributes of new technologies to treat diabetes and hypertension in our evidence-based clinical practice workshop. Then, we did focus group discussion and expert consultation on attributes and levels regarding reimbursement of new medical technologies. Fourteen experts (from six provinces in China) in reimbursement, health economics, healthcare service and evidence-based medicine were consulted. Criteria regarding needs of the technology (severity, benefit type of technology, unmet needs of reimbursed technology), comparative outcomes (effectiveness, safety/tolerability, patient-perceived/ patient-reported outcomes) and economic aspects of the technology (costs and cost-effectiveness) were required for health insurance reimbursement decisions. ${ }^{29}$ To better define the levels of attributes, we searched the famous health technology assessment database established by Canadian Agency for Drugs and Technologies in Health to select potential new technologies and find reasons for the recommendation of reimbursement. We found 68 reports regarding hypertension and diabetes which were published before March 2018. Data extraction form was developed and the attributes of new technologies were extracted. We further searched the database founded by
China National Medical Products Administration (NMPA, formerly China Food and Drug Administration or CFDA) according to the generic name of new technologies to see if they were licensed and available in China. We also referred to the list of medical technologies already covered by the public health insurance programmes in Jiangsu province. After completing the database search, we defined new medical technologies in this study as the therapeutics for hypertension and diabetes, which had been marketed in China but were not covered by the public health insurance programmes in Jiangsu in 2018. We determined the range of out-of-pocket costs according to the retail price of new technologies.

Attributes and levels of new medical technologies that were used in our research were listed in table 1. Details of the explanation of attributes and levels were shown in online supplemental appendix 1. Our purpose was to identify the specific technology attributes and levels that were preferred by patients, not the special technology used to treat a specific disease. Therefore, the scenarios in our DCE were not restricted to a particular type of disease.

\section{Experimental design and development of the questionnaire}

D-efficiency experimental design that maximised the precision of estimated choice-model parameters for a given number of choice questions ${ }^{30}$ was created by Ngene 1.1.2 software (ChoiceMetrics, Sydney, Australia). Prior coefficients were set to zero during the pilot. After obtaining priors of the attributes from the pilot, Bayesianefficient design was used to generate the final choice sets, which consisted of 30 pairs of scenarios and were divided into five blocks, with six pairs in each block. Blocking design promoted response efficiency by reducing the potential cognitive burden on respondents. ${ }^{31}$

We chose unlabelled over labelled DCE. Unlabelled DCE was widely used to investigate patients' preferences for treatment techniques. ${ }^{32-34}$ Respondents of unlabelled DCEs found that they were not subject to the psychological cues of the technology labels, thus reflecting the

Table 1 Attributes and levels of new medical technology in the DCE

\begin{tabular}{lll}
\hline Attributes & Levels & Variables coding \\
\hline Expected gains in health outcomes from the treatment & Not as expected; as expected & Binary \\
Likelihood of effective treatment & $30 \%$ to $90 \%$ & Continuous \\
Severity of target disease & Not severe; severe (but not fatal); fatal & Categorical \\
Incidence of serious adverse events & Often; occasionally; never or rarely & Categorical \\
Alternative technologies currently covered by insurance & Yes; no & Binary \\
Out-of-pocket cost per month (if not reimbursed) & CNY 300 to 3500 & Continuous \\
\hline
\end{tabular}

We defined new medical technologies according to research objectives. New medical technologies referred to new technologies that entered into the market recently before our study but were not included in the reimbursement lists of social health insurance schemes, such as URRBMI and UEBMI.

The average exchange rate of US $\$$ to CNY in 2018 was about 6.56 . Therefore, CNY 300 was approximately US $\$ 46$ and CNY 3500 was about US\$533.

CNY, Chinese yuan; DCE, discrete choice experiment; UEBMI, Urban Employees Basic Medical Insurance; URRBMI, Urban-Rural Residents Basic Medical Insurance. 
real-world choice situation. ${ }^{35}$ Also, in our research, new medical technologies to treat chronic diseases continue to emerge. Therefore, the unlabelled DCE was considered appropriate for our study. The forced-choice sets were used in our DCE because when no option had a definitive advantage, it was assumed that forced-choice under preference uncertainty would favour options that were easier to justify and associated with a lower likelihood of error and regret, such as compromise and asymmetrically dominant options. ${ }^{36}$

Examples of scenarios were shown in online supplemental appendix 2. Our final questionnaire contained two sections. Section A listed questions regarding participants' socio-demographic characteristics, past medical history, reasons for the hospital visit and health insurance information, dimensions and levels of EQ-5D-5L. EQ-5D-5L used a health-state classification system defining health in five dimensions, mobility, self-care, usual activities, pain/discomfort and anxiety/depression. Each of the five dimensions was divided into five levels of perceived problems, no problem, mild problems, moderate problems, severe problems and unable to/extreme problems. Section B was the DCE task.

\section{DCE implementation and data collection}

Our DCE was carried out from $15^{\text {th }}$ September to $15^{\text {th }}$ October 2018, in six tertiary public hospitals in four cities in Jiangsu province. Since our DCE aimed to inform decision-making on patient-centred care by identifying patients' preferences, the study sample consisted of patients with chronic diseases, not the general population. Due to the high prevalence, serious complications and the heavy burden of hypertension and diabetes, we selected patients with these diseases. Inclusion criteria were patients aged 18 years or older, participating in a social health insurance programme, with a history of diabetes or hypertension and taking medications regularly for more than 1 year. Patients were enrolled consecutively during the study period.

There was no general standard on the ideal sample size required for a DCE. ${ }^{37}$ Generally speaking, a less efficient design may also require a larger sample size, resulting in increased costs. ${ }^{38}$ Estimates of the sample size were usually determined based on previous research, rules of thumb and budget constraints. DCE studies showed that reliable models could be estimated in samples with more than 50 participants. ${ }^{39} 40$

The DCE questionnaires were administered through one-to-one, face-to-face interviews to ensure the validity and quality of the investigation. Our interviewers consisted of 13 medical students, all of whom were interns during the research period. For quality assurance, the interviewers were trained before the experiment. We compiled a survey training manual and asked each interviewer to give explanations to the scenarios. The interviewers were required to check whether the entire questionnaire was complete immediately after each interview. If any information was missing, they had to go back and ask patients to provide the information on site. For patients with blurred vision or illiteracy, the interviewers explained the meaning of the questionnaire item by item until the patients fully understood each item.

Verbal informed consent was obtained from all patients before both the pilot and the final survey. Patients were made aware that participation in the survey was voluntary. All data and information collected from patients was anonymous. During the pilot and formal survey, patients had to make a decision based on the assumption that only one technology could be covered due to limited health insurance funds. They were asked to think carefully and make a trade-off between two new medical technologies. The duration of the survey ranged from $20 \mathrm{~min}$ to 1 hour. We prepared a packed cotton towel for each patient as a gift (CNY 10 or US\$1.4). Patients were asked about how confident they felt in completing the choice sets. The confidence score ranged from 0 (not confident at all) to 10 (extremely confident) (online supplemental appendix 3 ). We excluded the DCE questionnaires with a confidence score of less than 8 .

\section{Patient and public involvement}

Ninety patients with diabetes or hypertension were engaged in the pilot survey to provide feedback on intelligibility, acceptability and reliability of the questionnaire. Response from the patients led to a more explicit and apprehensible description of the survey questions. The patients participating in the pilot were not included in the final survey. No patients were involved in the recruitment of study participants or the conduct of the study.

\section{Data analysis}

Our empirical analysis of the DCE data was based on the random utility model. Like previous research, ${ }^{26}$ we considered the utility, $\mathrm{U}$, that patient, $\mathrm{i}$, assigned to choice, $\mathrm{j}$, from J alternative choices, as the sum of two parts: observable component and unobservable component. The equation was developed as follows:

$$
\mathrm{U}_{\mathrm{ij}}=\mathrm{V}_{\mathrm{ij}}+\varepsilon_{\mathrm{ij}}=\beta_{0}+\beta_{1} \mathbf{x}_{1 \mathrm{ij}}+\beta_{2} \mathbf{x}_{2 \mathrm{ij}}+\ldots+\beta_{\mathrm{m}} \mathbf{x}_{\mathrm{mij}}+\varepsilon_{\mathrm{ij}}
$$

where $V_{\mathrm{ij}}$ was the observable component determined by patients' preferences for attributes $\left(x_{1}, \ldots, x_{m}\right), \varepsilon_{\mathrm{ij}}$ was the unobservable component of unobserved attributes and individual-level variations, and $\beta$ quantified the strength of preference for each attribute level. ${ }^{26}$

We implemented the above equation by mixed logit regression using Stata 14.2 SE (StataCorp LLC, College Station, Texas, USA) and was specified with 500 Halton draws. The mixed logit model allows for unknown heterogeneity in individual preferences and estimates both the mean preference weight and the standard deviation (SD). We assumed that all variables of the attributes, except for the constant, had a random component and that the weights of preference were normally distributed. The choice of patients was the dependent variable, and the selected technology attributes were independent variables. Dummy coding was used for categorical variables 
of our DCE data. For dummy variable coding, each model-estimated coefficient is a measure of the preference strength of that level relative to the omitted level of a specific attribute. ${ }^{41}{ }^{42}$ Subgroup analysis was performed by type of disease, type of insurance, HRQoL and gender. In each regression model, the attribute level with a negative coefficient indicates that patients would prefer not to move from the reference level to that level, while an attribute level with a positive coefficient indicates that patients would prefer to move from the reference level to that level. ${ }^{39}$

\section{RESULTS}

\section{Characteristics of patients}

A total of 410 patients were consented to participate in the DCE survey, and data from 408 patients were available for analysis with two patients excluded from the analysis due to non-compliance with the inclusion criteria, incomplete data and lack of confidence. The mean score for confidence was 8.80 (95\% CI 8.69 to 8.90), which suggested patients were confident in their choice. For details about numbers of patients in each sampled hospital, see online supplemental appendix 4 .

Table 2 presented the demographic and clinical characteristics of the included patients. The sample had more males than females $(53.92 \%$ vs $46.08 \%)$. The mean age of the patients was 62.34 years (ranging from 28 to 96 years). They were almost evenly split between UEBMI and URRBMI $(49.26 \%$ vs $50.74 \%)$. Most of the patients had hypertension $(63.97 \%)$ and $21.81 \%$ had diabetes, while $14.22 \%$ had both hypertension and diabetes. There was no statistically significant difference between hypertension and diabetes patients in terms of insurance types (UEBMI vs URRBMI, $\mathrm{p}=0.392$ ) and benefits (UEBMI with extra benefit vs UEBMI without extra benefit, $\mathrm{p}=0.359$ ) (online supplemental appendix 5). Cardiovascular disease (98 patients) was the most common comorbidity among 180 patients with chronic comorbid conditions other than hypertension and diabetes (online supplemental appendix 6 ).

\section{Regression analysis of the DCE data}

Our analysis found that the study patients highly valued the new technologies with never or rare incidence of serious adverse events $(\beta=0.884, p<0.01)$, followed by the expected gains in health outcomes from the treatment $(\beta=0.809, p<0.01) \quad($ table 3$)$. The likelihood of effective treatment was also a significant, positive predictor of patients' choice of new technologies $(\beta=0.455$, $\mathrm{p}<0.01)$ while out-of-pocket cost was a significant, negative predictor of patients' choice $(\beta=-0.258, \mathrm{p}<0.01)$. In contrast, whether there were alternative technologies currently covered by insurance did not seem to be an important consideration for the patients $(p>0.05)$. Unobservable preference heterogeneity as indicated by the estimated SD of the mean coefficients, were identified for four variables-expected gains in health outcomes from
Table 2 Characteristics of patients $(n=408)$

\begin{tabular}{lc} 
Characteristics & N (\%) \\
\hline $\begin{array}{l}\text { Gender } \\
\text { Male }\end{array}$ & $220(53.92)$ \\
Female & $188(46.08)$ \\
Age groups & \\
$18 \sim 45$ & $30(7.35)$ \\
$45 \sim 59$ & $131(32.11)$ \\
$60 \sim 74$ & $184(45.10)$ \\
$\quad \geq 75$ & $63(15.44)$ \\
Urban versus rural household registration \\
Urban \\
Rural & $210(51.47)$ \\
\end{tabular}

Education

$\begin{array}{lc}\text { Unschooled } & 39(9.56) \\ \text { Primary school } & 108(26.47) \\ \text { Junior high school } & 110(26.96) \\ \text { High school } & 89(21.81) \\ \text { Junior college or higher vocational } & 31(7.60) \\ \text { college } & \\ \text { Bachelor's degree or above } & 31(7.60) \\ \text { Employment } & \\ \text { Farmer } & 105(25.74) \\ \text { Urban employee } & 140(34.31) \\ \text { Retiree } & 112(27.45) \\ \text { Freelancers } & 32(7.84) \\ \text { Unemployed } & 19(4.66) \\ \text { Type of insurance } & \\ \text { UEBMI } & 201(49.26) \\ \text { URRBMI } & 207(50.74)\end{array}$

Family monthly income (CNY) $\dagger$

\begin{tabular}{lc}
$<2000$ & $83(20.34)$ \\
$2001 \sim 4000$ & $81(19.85)$ \\
$4001 \sim 6000$ & $93(22.79)$ \\
$6001 \sim 8000$ & $69(16.91)$ \\
$8001 \sim 10000$ & $41(10.05)$ \\
$>10000$ & $41(10.05)$ \\
\hline Type of patients & $83(20.34)$ \\
Outpatients & $325(79.66)$ \\
\hline Inpatients & \\
\hline Type of chronic diseases & $261(63.97)$ \\
Hypertension & $89(21.81)$ \\
Diabetes & $58(14.22)$ \\
Both & $180(44.12)$ \\
Comorbidities other than hypertension or diabetes \\
Yes & $228(55.88)$ \\
\hline No
\end{tabular}

Continued 
Table 2 Continued

\begin{tabular}{ll}
\hline Characteristics & N (\%) \\
\hline EQ-5D-5L index valuef & \\
$\leq 0.8$ & $127(31.13)$ \\
$>0.8$ & $281(68.87)$ \\
\hline
\end{tabular}

*One UEBMI patients and six URRBMI patients also enrolled in commercial health insurance.

†The average exchange rate between US dollars and Chinese yuan (CNY) in 2018 was 6.56.

$\ddagger$ The utility index was derived from the Chinese value sets. Currently, the well-accepted threshold of the EQ-5D-5L index value still lacks. However, in most cases, the EQ-5D-5L index value for patients with serious complications of diabetes and hypertension was equal to or less than 0.8 , as shown in studies conducted in China. ${ }^{20} 22 \mathrm{EQ}-5 \mathrm{D}-5 \mathrm{~L}$ index value $\leq 0.8$ group: median 0.6718 , IQR $-0.0818 \sim 0.7998$; EQ-5D-5L index value >0.8 group: median 0.9507, IQR $0.8410 \sim 1$.

UEBMI, Urban Employees Basic Medical Insurance; URRBMI, Urban-Rural Residents Basic Medical Insurance.

the treatment, the likelihood of effective treatment, outof-pocket cost and fatal disease.

\section{Subgroup analysis by type of disease}

Online supplemental appendix 7 presented the results from the subgroup analysis by type of disease (hypertension vs diabetes). While the two groups had similar results, there were two notable differences. One was that, although out-of-pocket cost remained a significant negative predictor, the coefficient for hypertension patients was $-0.178(p<0.05)$, not as important as for patients with diabetes $(\beta=-0.395, \mathrm{p}<0.01)$. The other was that the expected gains in health outcomes from the treatment seemed to be more important for diabetes patients $(\beta=$ $0.965, \mathrm{p}<0.01)$ when compared with those who only had hypertension $(\beta=0.716, p<0.01)$. The SD revealed coefficient heterogeneity in both subgroups for the random parameters of three variables-the likelihood of effective treatment, fatal disease and out-of-pocket cost.

\section{Subgroup analysis by type of insurance}

Online supplemental appendix 8 summarised the subgroup analyses by type of insurance. The expected gains in health outcomes from the treatment, likelihood of effective treatment and low incidence of serious adverse events were significant, positive predictors of technology choice $(\mathrm{p}<0.01)$ for both URRBMI and UEBMI patients. Whether there were alternative technologies currently covered by insurance was statistically insignificant for both groups ( $p>0.05$ ). However, these two groups differed remarkably in two technology attributes. The coefficient of out-of-pocket cost was significant for URRBMI patients $(\beta=-0.511, \mathrm{p}<0.01)$, but not for UEBMI patients $(\beta=$ $-0.071, \mathrm{p}>0.05)$. The severity of target disease also had significant coefficients for URRBMI patients $(p<0.01)$, but not for UEBMI patients.

We conducted further analysis of the UEBMI patients by excluding those UEBMI patients who enjoyed extra health insurance benefits. As indicated by Column (3), we found that out-of-pocket cost was a meaningful attribute for the remaining UEBMI patients $(\beta=-0.211$, $\mathrm{p}<0.05)$ although not as important as shown in URRBMI patients $(\beta=-0.211$ vs $\beta=-0.511)$. On the other hand, the severity of target disease remained statistically nonsignificant. Preference heterogeneity was identified for the lowest incidence of serious adverse events.

\section{Subgroup analysis by HRQoL}

Online supplemental appendix 9 demonstrated the results from the subgroup analysis by EQ-5D-5L index value, which was a valid measurement for HRQoL. The severity of target disease, both severe and fatal, was important for patients with an EQ-5D-5L index value less than or equal to $0.8(\mathrm{p}<0.01)$. However, it was statistically non-significant for patients with an EQ-5D-5L index value higher than $0.8(\mathrm{p}>0.05)$. Although patients' preferences for attributes including expected gains in health outcomes from the treatment, and lowest incidence of serious adverse events were statistically significant for both groups, they were less important as viewed by the group with the lower EQ-5D-5L index value. In patients

Table 3 DCE results from mixed logit model

\begin{tabular}{|c|c|c|}
\hline \multirow[b]{2}{*}{ Attributes } & \multicolumn{2}{|l|}{ All patients } \\
\hline & Mean (SE) & SD (SE) \\
\hline \multicolumn{3}{|c|}{$\begin{array}{l}\text { Expected gains in health outcomes from the treatment } \\
\text { Not as expected (reference) }\end{array}$} \\
\hline As expected & $0.809^{\star \star}(0.123)$ & $0.554^{\star}(0.275)$ \\
\hline $\begin{array}{l}\text { Likelihood of } \\
\text { effective treatment } \\
\text { (per } 10 \% \text { increase) }\end{array}$ & $0.455^{\star \star}(0.044)$ & $0.375^{\star \star}(0.055)$ \\
\hline
\end{tabular}

Severity of target disease

Not severe (reference)

\begin{tabular}{|c|c|c|}
\hline Severe & $0.291^{*}(0.123)$ & $0.316(0.431)$ \\
\hline Fatal & $0.208(0.147)$ & $1.264^{\star \star}(0.199)$ \\
\hline \multicolumn{3}{|c|}{$\begin{array}{l}\text { Incidence of serious adverse events } \\
\text { Often (reference) }\end{array}$} \\
\hline Occasionally & $0.575^{\star \star}(0.116)$ & $0.035(0.694)$ \\
\hline Never or rarely & $0.884^{\star \star}(0.142)$ & $0.900(0.206)$ \\
\hline
\end{tabular}

Alternative technologies currently covered by insurance Yes (reference)

\begin{tabular}{lcc}
\multicolumn{1}{c}{ No } & $0.087(0.104)$ & $0.095(0.501)$ \\
\hline $\begin{array}{l}\text { Out-of-pocket cost } \\
\text { (CNY 1000 per }\end{array}$ & $-0.258^{\star \star}(0.061)$ & $0.898^{\star \star}(0.090)$ \\
month increase) & & \\
Log likelihood & -1485.761 & \\
Participants & 408 & \\
Observations & 4896 & \\
\hline
\end{tabular}

SE, standard error; SD, standard deviation

$\mathrm{SD}$ estimates reflect preference heterogeneity in the participants. ${ }^{*} \mathrm{p}<0.05 ;{ }^{* *} \mathrm{p}<0.01$.

CNY, Chinese yuan; DCE, discrete choice experiment. 
with an EQ-5D-5L index value less than or equal to 0.8 , expected gains in health outcomes from the treatment had more variations than other attributes. However, the heterogeneity for the fatal disease was most significant in patients with an EQ-5D-5L index value greater than 0.8 .

Since the severity of target disease was an important attribute for URRBMI patients $(\mathrm{p}<0.01)$, but not for UEBMI patients. We did the $\chi^{2}$ test and results showed that the proportion of patients with a lower EQ-5D-5L index value was significantly higher in the URRBMI group $(\mathrm{p}<0.01)$ (online supplemental appendix 10).

\section{Subgroup analysis by gender}

We found that patients in both groups valued the new technologies with expected gains in health outcomes from the treatment, the likelihood of effective treatment, low incidence of serious adverse events and low out-ofpocket cost $(\mathrm{p}<0.01)$ (online supplemental appendix 11). However, the differences in preferences for attributes were not obvious between males and females.

\section{DISCUSSION}

\section{Summary of the findings}

Our study found that key technology attributes, including expected gains in health outcomes from the treatment, high likelihood of effective treatment and low incidence of serious adverse events were significant, positive predictors of patient choice for health insurance coverage. These results stand for the entire study sample and the subgroup analyses.

The out-of-pocket cost was a significant, negative predictor for the entire sample, showing that patients' preferences decreased as the out-of-pocket cost increased. We also found that out-of-pocket cost was a significant, negative predictor for both hypertension patients and diabetes patients, although it was less important for the former group than for the latter.

When it came to different insurance types, we identified preference heterogeneity as suggested by the previous DCE study. ${ }^{43}$ Specifically, we found that out-of-pocket cost was a significant, negative predictor for URRBMI patients' preference for insurance coverage, while the severity of target disease was a significant, positive predictor for this group of patients. But none of these attributes was a significant predictor for UEBMI patients. Our further analysis of the UEBMI patients by excluding those UEBMI patients who enjoyed extra health insurance benefits revealed that the remaining UEBMI patients regarded out-of-pocket cost as a significant, negative attribute for coverage, while the severity of target disease remained statistically non-significant.

Patients' HRQoL was measured and results suggested that patients with lower HRQoL tended to prefer new technologies that could have effects on severe or fatal diseases. The findings on the importance of disease severity regarding patients with lower HRQoL coincided with URRBMI patients. The reimbursement level and the hospitalisation rate of URRBMI patients were lower than that of UEBMI patients. ${ }^{15}$ Further analysis showed a relatively higher proportion of URRBMI patients with lower HRQoL. Also, our results have shown that gender is not a decisive factor for the preference of new technologies for reimbursement.

The degree to which respondent preferences were heterogeneous was described by the estimated SD around each mean preference estimate. Heterogeneity was found mainly for four variables-expected gains in health outcomes from the treatment, likelihood of effective treatment, out-of-pocket cost and fatal disease. Although heterogeneity existed, the preferences for new technologies with expected gains in health outcomes from the treatment, and the likelihood of effective treatment remained significant in all patients and each subgroup, suggesting that such attributes were generally valued by patients. Variations in preferences over out-of-pocket cost and fatal disease had implications for the optimal design of insurance reimbursement schemes and should be analysed in future studies.

\section{Comparison with other studies}

Our findings of patients valuing the effectiveness and safety of medical technologies were consistent with the results by prior studies from other countries which aimed to investigate patients' preferences for the treatment of chronic diseases. ${ }^{4-48}$ Our study confirmed that new technologies that could increase health benefits and minimise potential risks were preferred by patients.

However, variations in patients' preferences existed and mainly depended on patients' feelings of the disease. Previous research found that the median hospitalisation cost for patients with hypertension was lower than patients with diabetes ${ }^{49}{ }^{50}$ which supported our findings that out-of-pocket cost was not as important for hypertension patients as it was for diabetes patients.

We also identified preference heterogeneity among patients with different types of insurance. Although China's successful health insurance expansion over the past decade led to the country's universal health insurance coverage, variations in benefit coverage existed among different health insurance schemes, ${ }^{12}$ resulting in disparities in accessibility and affordability of medical services. $^{51}$ Such inequalities affected patients' preferences across different types of insurance. For example, we found that the out-of-pocket cost was a significant, negative predictor for URRBMI patients' preference, but not for all of the UEBMI patients. The finding reflected the fact that, compared with URRBMI, UEBMI had better benefits and a higher reimbursement level, especially for those UEBMI patients with extra benefits. The finding also fitted into the big picture of disparities across insurance schemes in China, as illustrated by prior research.

We found that URRBMI patients attached importance to the severity of the disease. We also found the association between lower HRQoL and preferences for technologies treating severe or fatal disease. Previous studies 
found that chronic disease patients with URRBMI had lower health service utilisation. ${ }^{52}$ Furthermore, URRBMI patients had significantly higher adjusted in-hospital mortality rates and shorter length of stay when compared with concurrent UEBMI patients. ${ }^{53} 54$ These findings suggested that a plausible explanation for the importance of disease severity for URRBMI patients might be mainly attributed to their perception of HRQoL and their anxiety about the potential severe or fatal consequences of chronic diseases.

\section{Implications of the study findings}

The rising prevalence of chronic diseases in China has major implications on its ability to provide timely, acceptable and affordable healthcare service for its citizens. To meet the demand for new medical technologies for treating chronic diseases, China's policymakers need to consider patients' preferences when deciding on insurance coverage for new medical technologies. Specifically, our findings that patients favoured new medical technologies with substantial health benefits and low risks suggested such technologies should be the priority of health insurance coverage. We suggest policymakers make evidence-based comparisons among technologies according to the attributes patients preferred.

Our findings that out-of-pocket cost was a major concern for URRBMI patients but not for all UEBMI patients suggested that policymakers need to make further efforts to reduce disparities in benefits and reimbursement levels between these two types of insurance and between UEBMI subgroups. The efforts will not only enhance financial protections for URRBMI patients and subgroups within UEBMI patients, but will also contribute to China's long-term goal of equalising benefits across insurance programmes. ${ }^{10}$

We found that patients did not care about alternative technologies currently covered by insurance. However, it is an essential attribute in reimbursement decisionmaking. Decision-makers need to compare the new technologies with available alternative technologies and to determine whether to cover new medical technologies or replace the alternatives. Policymakers and clinicians need to implement communication strategies to improve patients' awareness of the alternative therapies and reimbursement policies under the current insurance system to increase the appropriate use of the existing therapies.

\section{Strengths and limitations}

Our study used DCE to elicit preferences of chronic disease patients on insurance coverage of new medical technologies in China. We identified preference heterogeneity among patients with different types of insurance. Patients' HRQoL was measured, and the potential impact on preferences for reimbursement of new technologies was analysed. Our research helped to apply a patient-centred approach to policymaking and generated evidence that could inform insurance coverage decision-making.
Nevertheless, there are several limitations in our study. First, our samples were taken from tertiary hospitals in Jiangsu province. Patients receiving medical services from tertiary hospitals generally have serious and/or complex medical conditions. They have greater demand for healthcare services than other patients and may consider the issue of medical insurance coverage and reimbursement with caution. Jiangsu is an eastern, coastal province and one of the most economically developed regions in China. Future studies are needed to have a nationally representative sample by including patients at secondary and primary hospitals and, in particular, by including the economically underdeveloped regions in China.

Second, our study included patients with a history of diabetes or hypertension. Due to differences in nature and characteristics of the disease, the results may not represent the preferences of patients with other types of chronic diseases. Although prior DCEs ${ }^{44-48}$ made conclusions that were similar to ours in terms of the relative importance of technology attributes regarding benefits and risks. Future studies need to enrol patients with other diseases and conduct subgroup analyses to identify variations in patients' preferences across different types of diseases.

Third, there were only 43 UEBMI patients who enjoyed additional benefits of health insurance, and the limited sample size prevented us from conducting a separate analysis of this subgroup.

\section{CONCLUSION}

Chronic disease patients highly valued the health benefits and risks of new technologies, which are closely linked to their perceptions and feelings. Policymakers need to take new technologies with high therapeutic effectiveness and low risks for treating chronic diseases as a priority for health insurance coverage. More attempts should be made to reduce the gaps in benefits and reimbursement levels between insurance schemes to promote equitable access to healthcare services in China.

Acknowledgements We acknowledge the important contributions made by our interviewers who did one-to-one, face-to-face interviews with the patients. We are grateful to the patients for their time and efforts. We sincerely thank reviewers for providing valuable comments and suggestions on the manuscript. We acknowledge Caitlin Lupton for linguistic assistance. At the time of drafting the manuscript, Jinsong Geng was a fellow at the Fellowship in Health Policy and Insurance Research, Department of Population Medicine, Harvard Medical School and Harvard Pilgrim Healthcare Institute.

Contributors JG, XC and HY led the design and analysis of the discrete choice experiment. JG and $X C$ contributed to the literature search and data interpretation. $H B, D Q$ and $Y S$ contributed to implementing the discrete choice experiment. JG and $\mathrm{HY}$ performed the statistical analysis and wrote the manuscript.

Funding This work was supported by National Natural Science Foundation of China (Grant No. 71603138) and Jiangsu Overseas Visiting Scholar Program for University Prominent Young \& Middle-aged Teachers and Presidents.

Competing interests None declared.

Patient and public involvement Patients and/or the public were not involved in the design, or conduct, or reporting, or dissemination plans of this research.

Patient consent for publication Not required. 
Ethics approval This study, including the patient consent process, has been approved by the Medical Ethics Committee in Affiliated Hospitals of Nantong University (Ethical Approval-2016031) and conforms to the ethical guidelines of the Declaration of Helsinki.

Provenance and peer review Not commissioned; externally peer reviewed.

Data availability statement Data are available upon reasonable request. Data will be available upon reasonable request to the corresponding author.

Open access This is an open access article distributed in accordance with the Creative Commons Attribution Non Commercial (CC BY-NC 4.0) license, which permits others to distribute, remix, adapt, build upon this work non-commercially, and license their derivative works on different terms, provided the original work is properly cited, appropriate credit is given, any changes made indicated, and the use is non-commercial. See: http://creativecommons.org/licenses/by-nc/4.0/.

\section{ORCID iDs}

Jinsong Geng http://orcid.org/0000-0003-3389-9051

Hao Yu http://orcid.org/0000-0003-1091-9259

\section{REFERENCES}

1 World Health Organization. Global action plan for the prevention and control of noncommunicable diseases 2013-2020. Geneva: World Health Assembly, 2013: 5-9.

2 Lu J, Lu Y, Wang X, et al. Prevalence, awareness, treatment, and control of hypertension in China: data from 1.7 million adults in a population-based screening study (China peace million persons project). Lancet 2017;390:2549-58.

3 Wang L, Gao P, Zhang M, et al. Prevalence and ethnic pattern of diabetes and prediabetes in China in 2013. JAMA 2017;317:2515-23.

4 Adriaanse MC, Drewes HW, van der Heide I, et al. The impact of comorbid chronic conditions on quality of life in type 2 diabetes patients. Qual Life Res 2016;25:175-82.

5 Aubert CE, Fankhauser N, Marques-Vidal P, et al. Patterns of multimorbidity in internal medicine patients in Swiss university hospitals: a multicentre cohort study. Swiss Med Wkly 2019;149:w20094.

6 Wang J, Ma JJ, Liu J, et al. Prevalence and risk factors of comorbidities among hypertensive patients in China. Int $\mathrm{J} \mathrm{Med} \mathrm{Sci}$ 2017:14:201-12.

7 Bloom DE, Chen S, Kuhn M, et al. The economic burden of chronic diseases: estimates and projections for China, Japan, and South Korea. JEoA 2018:100163.

8 Yip W, Fu H, Chen AT, et al. 10 years of health-care reform in China: progress and gaps in universal health coverage. Lancet 2019;394:1192-204.

9 China's National Healthcare Security Administration. China healthcare security Diagnosis Related groups (CHS-DRG). Beijing, 2019: 44-6.

$10 \mathrm{Yu} \mathrm{H}$. Universal health insurance coverage for 1.3 billion people: what accounts for China's success? Health Policy 2015;119:1145-52.

$11 \mathrm{Li} \mathrm{Y,} \mathrm{Zhao} \mathrm{Y,} \mathrm{Yi} \mathrm{D,} \mathrm{et} \mathrm{al.} \mathrm{Differences} \mathrm{exist} \mathrm{across} \mathrm{insurance} \mathrm{schemes}$ in China post-consolidation. PLoS One 2017;12:e0187100.

12 Xiong X, Zhang Z, Ren J, et al. Impact of universal medical insurance system on the accessibility of medical service supply and affordability of patients in China. PLoS One 2018;13:e0193273.

13 Xian W, Xu X, Li J, et al. Health care inequality under different medical insurance schemes in a socioeconomically underdeveloped region of China: a propensity score matching analysis. BMC Public Health 2019;19:1373.

14 The State Council of the People's Republic of China. Opinion of the state Council on integrating the basic health insurance system for urban and rural residents, 2016. Available: http://www.gov.cn/ zhengce/content/2016-01/12/content_10582.htm

15 China's National Healthcare Security Administration. Statistical Bulletin of national social medical insurance development in 2018. Available: http://www.nhsa.gov.cn/art/2019/6/30/art 7 1477.html

16 General Office of the State Council of the People's Republic of China. Notice of the general office of the state Council on transmitting the opinions from the Ministry of labor and social security and the Ministry of finance on the implementation of medical subsidies for national civil servants, 2000. Available: http://www.gov.cn/gongbao/ content/2000/content 60249.htm

17 Wuxi No.2 People's Hospital. Medical insurance policy (the latest version of 2020), 2020. Available: https://www.wx2h.com/ywzc/ ywzcdetail/index/id/4/tid/6.html
18 Makovski TT, Schmitz S, Zeegers MP, et al. Multimorbidity and quality of life: systematic literature review and meta-analysis. Ageing Res Rev 2019;53:100903.

19 van Reenen M, Janssen B. EQ-5D-5L user guide: basic information on how to use the EQ-5D-5L instrument. Rotterdam: EuroQol Research Foundation, 2015.

20 Pan C-W, Sun H-P, Zhou H-J, et al. Valuing health-related quality of life in type 2 diabetes patients in China. Med Decis Making 2016;36:234-41.

21 Wang P, Luo N, Tai ES, et al. The EQ-5D-5L is more discriminative than the EQ-5D-3L in patients with diabetes in Singapore. Value Health Reg Issues 2016;9:57-62.

22 Liang Z, Zhang T, Lin T, et al. Health-Related quality of life among rural men and women with hypertension: assessment by the EQ-5D5L in Jiangsu, China. Qual Life Res 2019;28:2069-80.

23 Institute of Medicine Committee on Quality of Health Care in America. Crossing the quality chasm: a new health system for the 21st century. Washington, DC: The National Academies Press, 2001.

24 Lancsar E, Fiebig DG, Hole AR. Discrete choice experiments: a guide to model specification, estimation and software. Pharmacoeconomics 2017;35:697-716.

25 Clark MD, Determann D, Petrou S, et al. Discrete choice experiments in health economics: a review of the literature. Pharmacoeconomics 2014;32:883-902.

26 Ryan M, Kolstad J, Rockers P, et al. How to conduct a discrete choice experiment for health workforce recruitment and retention in remote and rural areas: a user guide with case studies. Geneva: World Health Organization Press, 2012.

27 Goetghebeur MM, Wagner M, Khoury H, et al. Evidence and Value: Impact on DEcisionMaking--the EVIDEM framework and potential applications. BMC Health Serv Res 2008;8:270.

28 Chen XW, Chen Q, Geng JS, et al. The application of multi-criteria decision analysis in medical insurance reimbursement: a systematic review. China Health Rev 2018;21:218-23.

29 Geng JS, Chen XW, XL Y, et al. Study on the evidence-based decision-making framework for reimbursement of new technologies in view of EVIDEM. Chinese $J$ Health Policy 2018;11:50-4.

30 Hensher DA, Rose JM, Greene WH. Applied choice analysis. 2nd ed. Cambridge: Cambridge University Press, 2015

31 Reed Johnson F, Lancsar E, Marshall D, et al. Constructing experimental designs for discrete-choice experiments: report of the ISPOR conjoint analysis experimental design good research practices Task force. Value Health 2013;16:3-13.

32 Nichols E, O'Hara NN, Degani Y, et al. Patient preferences for nutritional supplementation to improve fracture healing: a discrete choice experiment. BMJ Open 2018;8:e019685.

33 van Dijk JD, Groothuis-Oudshoorn CGM, Marshall DA, et al. An empirical comparison of discrete choice experiment and Best-Worst scaling to estimate stakeholders' risk tolerance for hip replacement surgery. Value Health 2016;19:316-22.

34 Bhattarai N, Mcmeekin P, Price $\mathrm{Cl}$, et al. Preferences for centralised emergency medical services: discrete choice experiment. BMJ Open 2019;9:e030966.

35 Kløjgaard ME, Bech M, Søgaard R. Designing a stated choice experiment: the value of a qualitative process. J Choice Model 2012;5:1-18.

36 Dhar R, Simonson I. The effect of forced choice on choice. JMR 2003:40:146-60.

37 de Bekker-Grob EW, Donkers B, Jonker MF, et al. Sample size requirements for discrete-choice experiments in healthcare: a practical guide. Patient 2015;8:373-84

38 Vanniyasingam T, Cunningham CE, Foster G, et al. Simulation study to determine the impact of different design features on design efficiency in discrete choice experiments. BMJ Open 2016;6:e011985.

39 Oluboyede Y, Ternent L, Vale L, et al. Using a discrete-choice experiment to estimate the preferences of clinical practitioners for a novel non-invasive device for diagnosis of peripheral arterial disease in primary care. Pharmacoecon Open 2019;3:571-81.

40 Shanahan M, Seddon J, Ritter A, et al. Valuing families' preferences for drug treatment: a discrete choice experiment. Addiction 2020;115:690-9.

41 Hauber AB, González JM, Groothuis-Oudshoorn CGM, et al. Statistical methods for the analysis of discrete choice experiments: a report of the ISPOR conjoint analysis good research practices Task force. Value Health 2016;19:300-15.

42 Kløjgaard ME, Manniche C, Pedersen LB, et al. Patient preferences for treatment of low back pain-a discrete choice experiment. Value Health 2014;17:390-6. 
43 de Bekker-Grob EW, Swait JD, Kassahun HT, et al. Are healthcare choices predictable? the impact of discrete choice experiment designs and models. Value Health 2019;22:1050-62.

44 Bröckelmann PJ, McMullen S, Wilson JB, et al. Patient and physician preferences for first-line treatment of classical Hodgkin lymphoma in Germany, France and the United Kingdom. Br J Haematol 2019:184:202-14

45 Forsander G, Stallknecht S, Samuelsson U, et al. Preferences for treatment among adolescents with type 1 diabetes: a national study using a discrete choice experiment model. Diabet Med 2018;35:621-9.

46 Havrilesky LJ, Lim S, Ehrisman JA, et al. Patient preferences for maintenance PARP inhibitor therapy in ovarian cancer treatment. Gynecol Oncol 2020;156:561-7.

47 Ho K-A, Acar M, Puig A, et al. What do Australian patients with inflammatory arthritis value in treatment? a discrete choice experiment. Clin Rheumatol 2020;39:1077-89.

48 Thieu VT, Robinson S, Kennedy-Martin T, et al. Patient preferences for glucagon-like peptide 1 receptor-agonist treatment attributes. Patient Prefer Adherence 2019;13:561-76.
49 Mutowo MP, Lorgelly PK, Laxy M, et al. The hospitalization costs of diabetes and hypertension complications in Zimbabwe: estimations and correlations. J Diabetes Res 2016;2016:9754230

50 Rosa MQM, Rosa RDS, Correia MG, et al. Disease and economic burden of hospitalizations attributable to diabetes mellitus and its complications: a nationwide study in Brazil. Int J Environ Res Public Health 2018;15:E294.

51 Zhao C, Wang C, Shen C, et al. China's achievements and challenges in improving health insurance coverage. Drug Discov Ther 2018;12:1-6.

52 Xie X, Wu Q, Hao Y, et al. Identifying determinants of socioeconomic inequality in health service utilization among patients with chronic non-communicable diseases in China. PLoS One 2014:9:e100231.

$53 \mathrm{Chen} \mathrm{H}$, Shi L, Xue M, et al. Geographic variations in In-Hospital mortality and use of percutaneous coronary intervention following acute myocardial infarction in China: a nationwide Cross-Sectional analysis. J Am Heart Assoc 2018;7:e008131.

54 Lin X, Cai M, Tao H, et al. Insurance status, inhospital mortality and length of stay in hospitalised patients in Shanxi, China: a crosssectional study. BMJ Open 2017;7:e015884. 